\title{
On Certain Matrices of Bernoulli Numbers
}

\author{
Ruiming Zhang' and Li-Chen Chen ${ }^{2}$ \\ ${ }^{1}$ The Institute of Applied Mathematics, College of Science, Northwest A\&F University, Yangling, Shaanxi 712100, China \\ ${ }^{2}$ Department of Financial Engineering and Actuarial Mathematics, Soochow University, 56 Kueiyang Street, Sec. 1, Taipei 100, Taiwan
}

Correspondence should be addressed to Ruiming Zhang; ruimingzhang@yahoo.com

Received 3 May 2014; Revised 16 July 2014; Accepted 24 July 2014; Published 5 August 2014

Academic Editor: Guo-Cheng Wu

Copyright (C) 2014 R. Zhang and L.-C. Chen. This is an open access article distributed under the Creative Commons Attribution License, which permits unrestricted use, distribution, and reproduction in any medium, provided the original work is properly cited.

In this work we compute the determinant and inverse matrices for a certain symmetric matrix of Rayleigh sums. As a special case we also obtain the determinants and inverses for the matrices of the Bernoulli numbers and related numbers.

\section{Introduction}

The sequence of Bernoulli numbers $B_{n}$ is one of the most important sequences in mathematics. It has deep connections to number theory, for instance, the Bernoulli numbers are used to express the values of $\zeta(2 n)$, where $\zeta(s)$ is the Riemann zeta function and $n$ is a positive integer $[1,2]$. The Bernoulli numbers are also very important in analysis, for example, they appear in the Euler-Maclaurin formula [1], which is very important in mathematics and physics. The Bernoulli numbers are also very important in asymptotics of $q$-special functions; for example, in [3] we proved a complete asymptotic expansion of $q$-Gamma function $\Gamma_{q}(z)$ on the complex plane in terms of Bernoulli polynomials and Bernoulli polynomials. The applications of Bernoulli numbers in applied mathematics are just too many to list all of them; just to name a few, for example, see [4-6]. The Rayleigh sums $\sigma_{v}^{(n)}$ generalize $\zeta(2 n)$ and it is known that $\sigma_{1 / 2}^{(n)}$ is a rational multiple of $B_{2 n}$ [7]. In this work we first derive the inverse and determinant of a certain symmetric matrix defined by $\sigma_{v}^{(n)}$ and then specialize the result to the matrices defined by Bernoulli numbers $B_{n}$ and related numbers $S_{n}$.

But we have to emphasize that the present work demonstrated a method to compute inverses of certain Hankel matrices, not just determinants. In fact there are many known methods to compute determinants; for example, see $[1,8-11]$.

\section{Preliminaries}

For $v>-1$ the Bessel function of first kind is defined by $[1,7$, 11, 12]:

$$
J_{\nu}(z)=\frac{1}{\Gamma(\nu+1)} \sum_{k=0}^{\infty} \frac{(-1)^{k}}{k !(\nu+1)_{k}}\left(\frac{z}{2}\right)^{2 k+v},
$$

where

$$
\begin{aligned}
& \frac{1}{\Gamma(a)}=a \prod_{j=1}^{\infty}\left(1+\frac{a}{j}\right)\left(1+\frac{1}{j}\right)^{-a}, \\
& (a)_{n}=\frac{\Gamma(a+n)}{\Gamma(a)}, \quad n \in \mathbb{Z}, a \in \mathbb{C} .
\end{aligned}
$$

As a special case we have

$$
J_{1 / 2}(z)=\sqrt{\frac{2}{\pi z}} \sin z .
$$

It is known that the even entire function $J_{\nu}(z) z^{-v}$ has infinitely many zeros, all of which are real. Let

$$
0<j_{v, 1}<j_{v, 2}<\cdots
$$

be all its positive zeros; then the Rayleigh sum is defined by [7]

$$
\sigma_{v}^{(n)}=\sum_{k=1}^{\infty} \frac{1}{j_{v, k}^{2 n}}, \quad n \in \mathbb{N} .
$$


Clearly [1],

$$
\sigma_{1 / 2}^{(n)}=\sum_{n=1}^{\infty} \frac{1}{(n \pi)^{2 k}}=\frac{(-1)^{n+1} 2^{2 n-1} B_{2 n}}{(2 n) !},
$$

where the Bernoulli numbers $B_{n}$ are defined by $[1,2,12]$

$$
\frac{z}{e^{z}-1}=\sum_{n=0}^{\infty} B_{n} \frac{z^{n}}{n !}, \quad|z|<2 \pi .
$$

The related numbers $\left\{S_{n}\right\}_{n=1}^{\infty}$ are defined by $[2,13]$

$$
S_{n}=2\left(\frac{2}{\pi}\right)^{n} \sum_{k=-\infty}^{\infty} \frac{1}{(4 k+1)^{n}}
$$

for $n=2,3, \ldots$ and $S_{1}=1$; it is known that

$$
\frac{(-1)^{n+1} B_{2 n}}{(2 n) !}=\frac{S_{2 n}}{2^{2 n}\left(2^{2 n}-1\right)}, \quad n \in \mathbb{N} \text {. }
$$

\section{Main Results}

Theorem 1. Given a nonnegative integer $n$, one has

$$
\begin{gathered}
\operatorname{det}\left(\sigma_{\nu}^{(j+k+1)}\right)_{j, k=1}^{n}=\frac{2^{(n+1)(2 n+1)}}{((\nu+1) / 2)_{n+1} \prod_{k=0}^{n}(\nu+1)_{2 k}^{2}}, \\
\left\{\left(\sigma_{\nu}^{(j+k+1)}\right)_{j, k=1}^{n}\right\}^{-1} \\
=\left\{\sum _ { m = 0 } ^ { n } \left((-1)^{j+k}(2 m+\nu+1)(m+j) !(m+k) !\right.\right. \\
\quad \times(\nu+1)_{m+j}(\nu+1)_{m+k} \\
\quad \times\left(4^{j+k+1}(2 j) !(2 k) !(m-j) !(m-k) !\right. \\
\left.\left.\left.\times(\nu+1)_{m-j}(\nu+1)_{m-k}\right)^{-1}\right)\right)_{j, k=0}^{n}
\end{gathered}
$$

for $v>-1$.

Corollary 2. For any nonnegative integer $n$, one has

$$
\begin{gathered}
\operatorname{det}\left(\frac{B_{2 j+2 k+2}}{(2 j+2 k+2) !}\right)_{j, k=1}^{n}=\frac{1}{(3 / 4)_{n+1} \prod_{k=0}^{n}(3 / 2)_{2 k}^{2}}, \\
\left\{\left(\frac{B_{2(j+k+1)}}{(2 j+2 k+2) !}\right)_{j, k=0}^{n}\right\}^{-1} \\
=\left(\sum _ { m = 0 } ^ { n } \left(\left(m+\frac{3}{4}\right)(m+j) !(m+k) !\left(\frac{3}{2}\right)_{m+j}\left(\frac{3}{2}\right)_{m+k}\right.\right. \\
\times\left((2 j) !(2 k) !(m-j) !(m-k) !\left(\frac{3}{2}\right)_{m-j}\right. \\
\left.\left.\left.\times\left(\frac{3}{2}\right)_{m-k}\right)^{-1}\right)\right)_{j, k=0}^{n},
\end{gathered}
$$

or, equivalently,

$$
\begin{gathered}
\operatorname{det}\left(\frac{S_{2 j+2 k+2}}{4^{j+k+1}-1}\right)_{j, k=0}^{n}=\frac{4^{(n+1)^{2}}}{(3 / 4)_{n+1} \prod_{k=0}^{n}(3 / 2)_{2 k}^{2}} \\
\left\{\left(\frac{S_{2 j+2 k+2}}{4^{j+k+1}-1}\right)_{j, k=0}^{n}\right\}^{-1} \\
=\left(\sum _ { m = 0 } ^ { n } \left(\left(m+\frac{3}{4}\right)(m+j) !(m+k) !\left(\frac{3}{2}\right)_{m+j}\left(\frac{3}{2}\right)_{m+k}\right.\right. \\
\times\left((-4)^{j+k}(2 j) !(2 k) !(m-j) !(m-k) !\right. \\
\left.\left.\left.\times\left(\frac{3}{2}\right)_{m-j}\left(\frac{3}{2}\right)_{m-k}\right)^{-1}\right)\right)_{j, k=0}^{n} .
\end{gathered}
$$

\section{Proofs}

Given a probability measure $d \mu(x)$ on $\mathbb{R}$ such that $\int_{\mathbb{R}} x^{2 n} d \mu(x)<\infty$ for all $n \in \mathbb{R}$, we define the inner product for $d \mu(x)$ square integrable functions $f(x)$ and $g(x)$ by

$$
(f, g)=\int_{-\infty}^{\infty} f(x) g(x) d \mu(x) .
$$

For each $n \in \mathbb{N} \cup\{0\}$, let $G_{n}=\left(m_{j, k}\right)_{j, k=0}^{n}$ with $m_{j, k}=$ $\left(u_{j}, u_{k}\right)$ for $j, k=0,1, \ldots, n$ where $\left\{u_{k}(x)\right\}_{k=0}^{\infty}$ is a sequence of polynomials with $u_{0}(x)=1$ such that, for each $n,\left\{u_{k}(x)\right\}_{k=0}^{n}$ are linearly independent. Then there is a unique orthonormal system $\left\{p_{k}(x)\right\}_{k=0}^{\infty}[1,10,11]$ :

$$
\begin{aligned}
p_{n}(x)= & \frac{1}{\sqrt{\operatorname{det} G_{n} \operatorname{det} G_{n-1}}} \\
& \times \operatorname{det}\left(\begin{array}{ccccc}
m_{0,0} & m_{0,1} & m_{0,2} & \cdots & m_{0, n} \\
m_{1,0} & m_{1,1} & m_{1,2} & \cdots & m_{1, n} \\
\vdots & \vdots & \vdots & \ddots & \vdots \\
m_{n-1,0} & m_{n-1,1} & m_{n-1,2} & \cdots & m_{n-1, n} \\
u_{0}(x) & u_{1}(x) & u_{2}(x) & \cdots & u_{n}(x)
\end{array}\right),
\end{aligned}
$$

with positive leading coefficient in $u_{n}(x)$. Clearly we have $p_{n}(x)=\sum_{j=0}^{n} a_{n, j} u_{j}(x)$ for some real numbers $a_{j, k}$ for $j, k=$ $0,1, \ldots, n$ and $a_{j, k}=0$ for $k>j$.

Lemma 3. For each nonnegative integer $n$, let $G_{n}=\left(m_{j, k}\right)_{j, k=0}^{n}$ and $A_{n}=\left(a_{j, k}\right)_{j, k=0}^{n}$. Then

$$
\operatorname{det} G_{n}=\prod_{j=0}^{n} a_{j, j}^{-2}, \quad G_{n}^{-1}=A_{n}^{T} A_{n} .
$$


Proof. From (15) and $p_{n}(x)=\sum_{j=0}^{n} a_{n, j} u_{j}(x)$ it is clear that

$$
a_{n, n}=\sqrt{\frac{\operatorname{det} G_{n-1}}{\operatorname{det} G_{n}}}, \quad \operatorname{det} G_{n}=\prod_{j=0}^{n} a_{j, j}^{-2} .
$$

For each $n$, since both $\left\{p_{k}(x)\right\}_{k=0}^{n}$ and $\left\{u_{k}(x)\right\}_{k=0}^{n}$ are a basis for the same set of polynomials, $A_{n}$ must be invertible for each $n \in \mathbb{N} \cup\{0\}$. We denote $A_{n}^{-1}=\left(s_{j, k}\right)_{j, k=0}^{n}$; then $u_{j}(x)=$ $\sum_{\ell=0}^{n} s_{j, \ell} p_{\ell}(x)$ for $j=0,1, \ldots, n$. Clearly, $s_{j, \ell}=0$ for $\ell>j$. Thus,

$$
m_{j, k}=\left(u_{j}(x), u_{k}(x)\right)=\sum_{m=0}^{n} s_{j, m} s_{k, m}
$$

for $j, k=0,1, \ldots, n$, which is

$$
G_{n}=A_{n}^{-1}\left(A_{n}^{-1}\right)^{T}=A_{n}^{-1}\left(A_{n}^{T}\right)^{-1}
$$

and hence $G_{n}^{-1}=A_{n}^{T} A_{n}$.

4.1. Proof of Theorem 1. The normalized even order Lommel polynomials are defined by [11]

$$
\begin{aligned}
h_{n}(x) & =\frac{\sqrt{2 n+v+1}}{2} h_{2 n, v+1}(x) \\
& =\sum_{k=0}^{n} \frac{(-1)^{n-k} \sqrt{2 n+v+1}(n+k) !(\nu+1)_{n+k}}{2^{2 k+1}(2 k) !(n-k) !(v+1)_{n-k}}\left(x^{2}\right)^{k},
\end{aligned}
$$

for $n \in \mathbb{N}$ and $h_{0}(x)=\sqrt{v+1} / 2$. They satisfy the orthogonal relation

$$
\sum_{k=1}^{\infty} \frac{1}{j_{v, k}^{2}} h_{m}\left(\frac{1}{j_{v, k}}\right) h_{n}\left(\frac{1}{j_{v, k}}\right)=\delta_{m, n} .
$$

For $n=0,1, \ldots$, it is clear that the $n$th moment with respect to the measure of orthogonality is

$$
m_{n}=\sum_{k=1}^{\infty} \frac{1}{j_{v, k}^{2+2 n}}=\sigma_{v}^{(n+1)}
$$

Let $u_{n}(x)=x^{2 n}$ for $n=0,1, \ldots$; then

$$
a_{n, k}=\frac{(-1)^{n-k} \sqrt{2 n+\nu+1}(n+k) !(\nu+1)_{n+k}}{2^{2 k+1}(2 k) !(n-k) !(\nu+1)_{n-k}} .
$$

By Lemma 3, the matrix $\left(\sigma_{\nu}^{(j+k+1)}\right)_{j, k=0}^{n}$ has determinant

$$
\prod_{k=0}^{n} \frac{2^{4 k+2}}{(\nu+1+2 k)(\nu+1)_{2 k}^{2}}=\frac{2^{(n+1)(2 n+1)}}{((\nu+1) / 2)_{n+1} \prod_{k=0}^{n}(\nu+1)_{2 k}^{2}}
$$

and its inverse $\left(\gamma_{j, k}\right)_{j, k=0}^{n}$ has elements

$$
\begin{aligned}
\gamma_{j, k}=\sum_{m=0}^{n}( & (-1)^{j+k}(2 m+v+1)(m+j) !(m+k) ! \\
& \times(\nu+1)_{m+j}(\nu+1)_{m+k} \\
& \times\left(4^{j+k+1}(2 j) !(2 k) !(m-j) !(m-k) !\right. \\
& \left.\left.\times(\nu+1)_{m-j}(\nu+1)_{m-k}\right)^{-1}\right) .
\end{aligned}
$$

4.2. Proof of Corollary 2. From (24), (25), and (6), we get

$$
\begin{aligned}
& \operatorname{det}\left(\frac{(-1)^{j+k} 2^{2 j+2 k+1} B_{2(j+k+1)}}{(2 j+2 k+2) !}\right)_{j, k=0}^{n} \\
& =\frac{2^{(n+1)(2 n+1)}}{(3 / 4)_{n+1} \prod_{k=0}^{n}(3 / 2)_{2 k}^{2}},
\end{aligned}
$$

$$
\begin{gathered}
\left\{\left(\frac{(-1)^{j+k} 2^{2 j+2 k+1} B_{2(j+k+1)}}{(2 j+2 k+2) !}\right)_{j, k=0}^{n}\right\}^{-1} \\
=\left(\sum _ { m = 0 } ^ { n } \left((-1)^{j+k}\left(2 m+\frac{3}{2}\right)(m+j) !(m+k) !\right.\right. \\
\times\left(\frac{3}{2}\right)_{m+j}\left(\frac{3}{2}\right)_{m+k} \\
\times\left(4^{j+k+1}(2 j) !(2 k) !(m-j) !(m-k) !\left(\frac{3}{2}\right)_{m-j}\right. \\
\left.\left.\left.\quad \times\left(\frac{3}{2}\right)_{m-k}\right)^{-1}\right)\right)_{j, k=0}^{n} .
\end{gathered}
$$

They are simplified to

$$
\begin{gathered}
\operatorname{det}\left(\frac{B_{2(j+k+1)}}{(2 j+2 k+2) !}\right)=\frac{1}{(3 / 4)_{n+1} \prod_{k=0}^{n}(3 / 2)_{2 k}^{2}} \\
\left\{\left(\frac{B_{2(j+k+1)}}{(2 j+2 k+2) !}\right)_{j, k=0}^{n}\right\}^{-1} \\
=\left(\sum _ { m = 0 } ^ { n } \left(\left(m+\frac{3}{4}\right)(m+j) !(m+k) !\left(\frac{3}{2}\right)_{m+j}\left(\frac{3}{2}\right)_{m+k}\right.\right. \\
\times\left((2 j) !(2 k) !(m-j) !(m-k) !\left(\frac{3}{2}\right)_{m-j}\right. \\
\left.\left.\left.\times\left(\frac{3}{2}\right)_{m-k}\right)^{-1}\right)\right)_{j, k=0}^{n} .
\end{gathered}
$$


By (9) we get

$$
\begin{gathered}
\operatorname{det}\left(\frac{S_{2 j+2 k+2}}{2\left(4^{j+k+1}-1\right)}\right)_{j, k=0}^{n}=\frac{2^{(n+1)(2 n+1)}}{(3 / 4)_{n+1} \prod_{k=0}^{n}(3 / 2)_{2 k}^{2}} \\
\left\{\left(\frac{S_{2 j+2 k+2}}{2\left(4^{j+k+1}-1\right)}\right)_{j, k=0}^{n}\right\} \\
=\left(\sum _ { m = 0 } ^ { n } \left((-1)^{j+k}\left(2 m+\frac{3}{2}\right)(m+j) !(m+k) !\left(\frac{3}{2}\right)_{m+j}\right.\right. \\
\times\left(\frac{3}{2}\right)_{m+k} \\
\times\left(4^{j+k+1}(2 j) !(2 k) !(m-j) !(m-k) !\left(\frac{3}{2}\right)_{m-j}\right. \\
\left.\left.\left.\times\left(\frac{3}{2}\right)_{m-k}\right)^{-1}\right)\right)_{j, k=0}^{n},
\end{gathered}
$$

which are simplified to (12) and (13), respectively.

\section{Conflict of Interests}

The authors declare that there is no conflict of interests regarding the publication of this paper.

\section{Acknowledgments}

The first and corresponding author of this work, Ruiming Zhang, is partially supported by the National Natural Science Foundation of China, Grant no. 11371294. He also thanks Professors Jyh-Hao Lee, Derchyi Wu, for their hospitalities during his visits to Institute of Mathematics, Academia Sinica, Taipei.

\section{References}

[1] G. E. Andrews, R. Askey, and R. Roy, Special Functions, vol. 71 of Encyclopedia of Mathematics and its Applications, Cambridge University Press, Cambridge, UK, 1999.

[2] Wikipedia, http://en.wikipedia.org/wiki/Bernoulli_number.

[3] R. Zhang, "On asymptotics of the \$q\$-exponential and \$q\$gamma functions," Journal of Mathematical Analysis and Applications, vol. 411, no. 2, pp. 522-529, 2014.

[4] E. Tohidi and F. Toutounian, "Convergence analysis of Bernoulli matrix approach for one-dimensional matrix hyperbolic equations of the first order," Computers \& Mathematics with Applications, vol. 68, no. 1-2, pp. 1-12, 2014.

[5] F. Toutounian, E. Tohidi, and S. Shateyi, "A collocation method based on the Bernoulli operational matrix for solving highorder linear complex differential equations in a rectangular domain," Abstract and Applied Analysis, vol. 2013, Article ID 823098, 12 pages, 2013.

[6] F. Toutounian and E. Tohidi, "A new Bernoulli matrix method for solving second order linear partial differential equations with the convergence analysis," Applied Mathematics and Computation, vol. 223, pp. 298-310, 2013.
[7] G. N. Watson, A Treatise on the Theory of Bessel Functions, Cambridge University Press, New York, NY, USA, 2nd edition, 1944.

[8] W. A. Al-Salam and L. Carlitz, "Some determinants of Bernoulli, Euler and related numbers," Portugaliae Mathematica, vol. 18, pp. 91-99, 1959.

[9] C. Krattenthaler, "Advanced determinant calculus," Séminaire Lotharingien de Combinatoire, vol. 42, article B42q, 67 pages, 1999.

[10] T. S. Chihara, An Introduction to Orthogonal Polynomials, Gordon and Breach, New York, NY, USA, 1978.

[11] M. E. H. Ismail, Continuous and Discrete Orthogonal Polynomials, Cambridge University Press, Cambridge, UK, 2005.

[12] R. Zhang, "Sums of zeros for certain special functions," Integral Transforms and Special Functions, vol. 21, no. 5-6, pp. 351-365, 2010.

[13] N. D. Elkies, "On the sums $\sum_{k=-\infty}^{\infty}(4 k+1)^{-n}$ ", The American Mathematical Monthly, vol. 110, no. 7, pp. 561-573, 2003. 


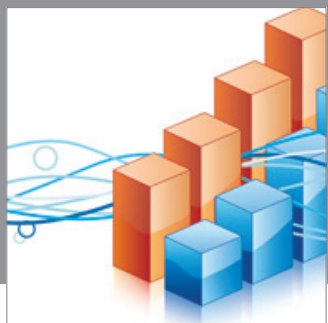

Advances in

Operations Research

mansans

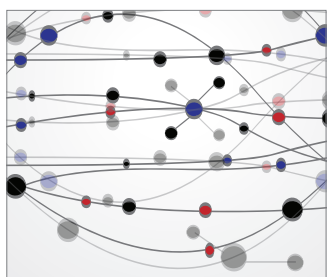

The Scientific World Journal
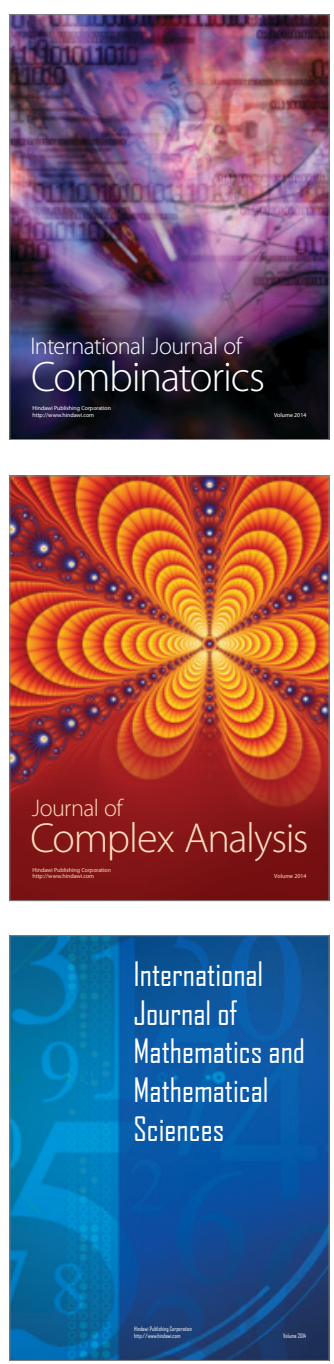
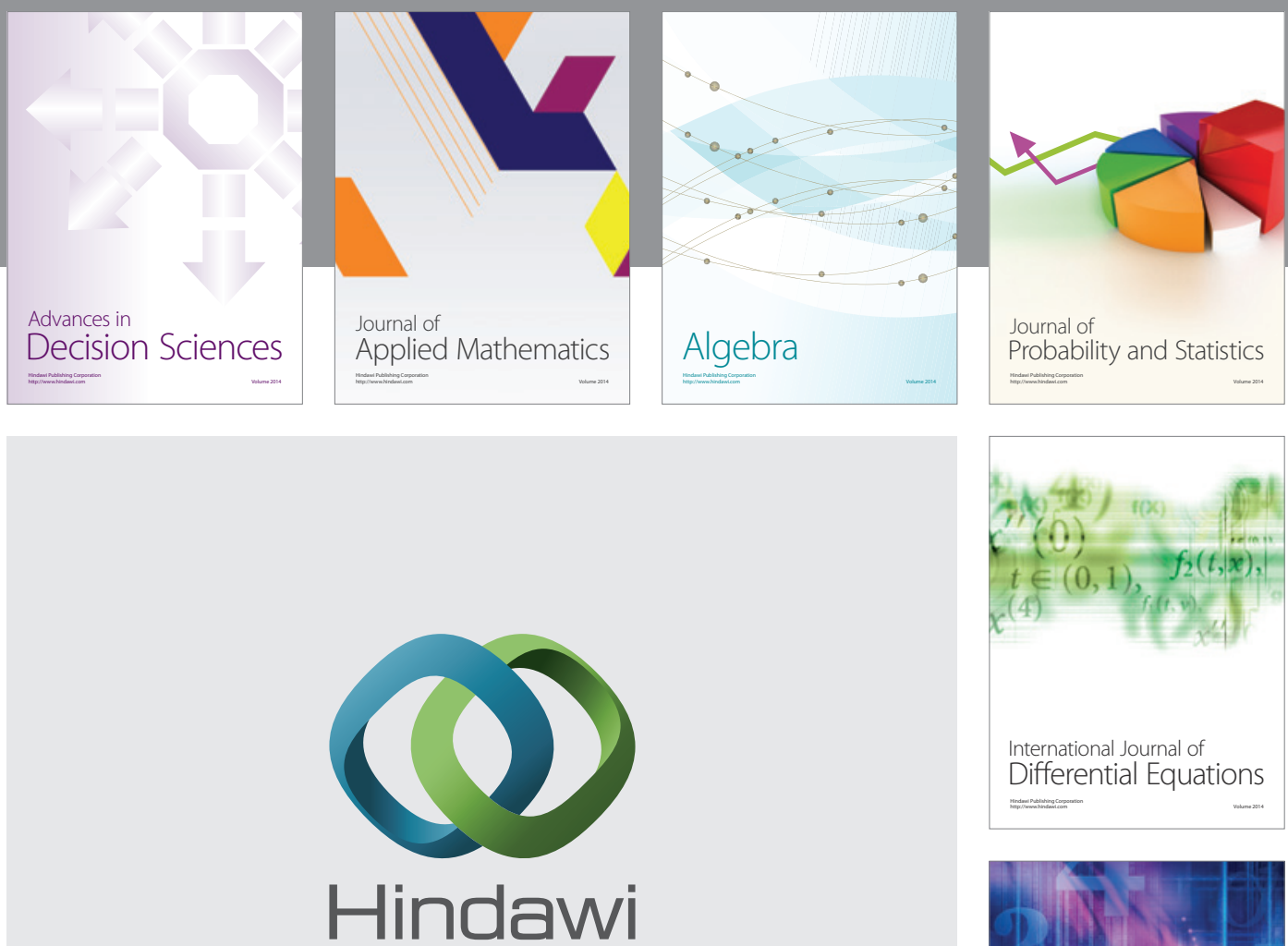

Submit your manuscripts at http://www.hindawi.com
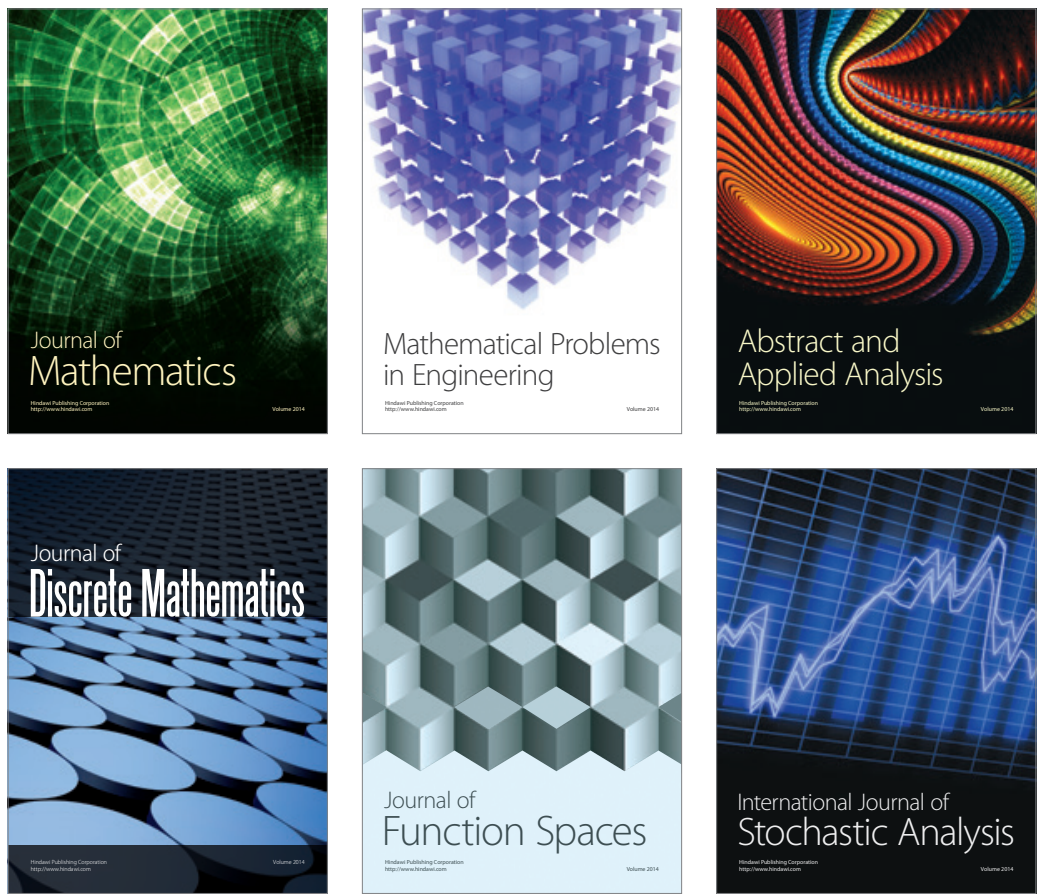

Journal of

Function Spaces

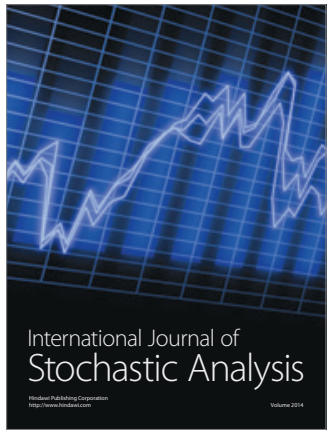

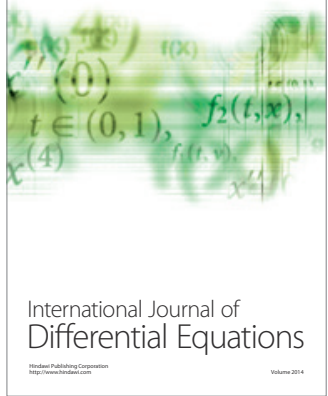
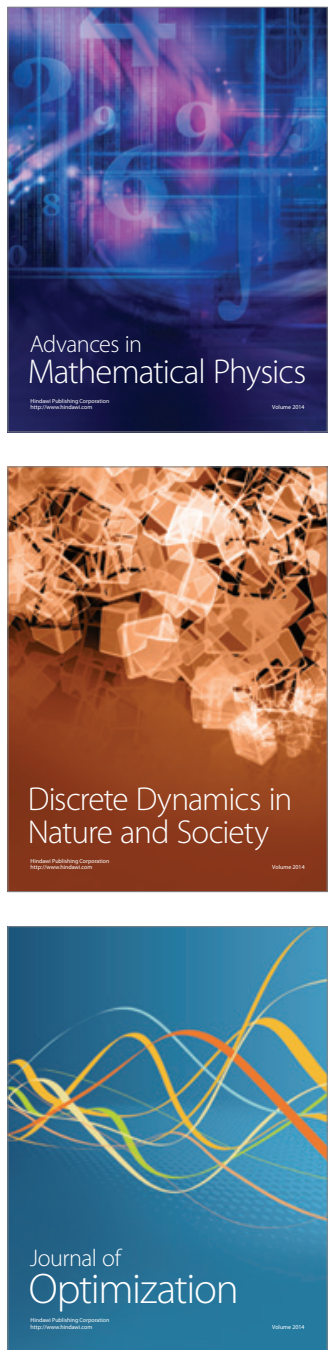